\title{
Coherent Ray Tracing Simulation Of Non-Imaging Laser Beam Shaping With Multi-Aperture Elements
}

\author{
Raoul Kirner ${ }^{1, *}$, Wilfried Noell $^{1}$, Toralf Scharf ${ }^{2}$, and Reinhard Voelkel $^{1}$ \\ ${ }^{1}$ SUSS MicroOptics SA, CH-2068 Hauterive, Switzerland \\ ${ }^{2}$ NAM, École Polytechnique Fédérale de Lausanne (EPFL), CH-1015 Lausanne, Switzerland
}

\begin{abstract}
The application of laser light sources for illumination tasks like in mask aligner lithography relies on non-imaging optical systems with multi-aperture elements for beam shaping. When simulating such systems, the traditional approach is to separate the beam-shaping part (incoherent simulation) from dealing with coherence properties of the illuminating laser light source (diffraction theory with statistical treatment). We present an approach using Gaussian beam decomposition to include coherence simulation into ray tracing, combining these two parts, to get a complete picture in one simulation. We discuss source definition for such simulations, and verify our assumptions on a well-known system. We then apply our approach to an imaging beam shaping setup with microoptical multi-aperture elements. We compare the simulation to measurements of a similar beam-shaping setup with a $193 \mathrm{~nm}$ continuous-wave laser in a mask-aligner configuration.
\end{abstract}

\section{Introduction}

To improve the resolution in proximity mask aligner lithography, decreasing the exposure wavelength $\lambda$ is the only way if a certain exposure gap $g$ is to be maintained as resolution is given by [1]:

$$
\text { Resolution } \sim \sqrt{g \cdot \lambda} .
$$

This requires light sources emitting at shorter wavelengths than the classically used $\mathrm{Hg}$ high-pressure arc lamps, like excimer lasers or TOPTICA's continuouswave $193 \mathrm{~nm}$ diode laser light source [2].

Mask aligner lithography relies on uniform illumination of the photomask, containing the structural information which is to be transmitted to a photoresistcoated substrate. Uniform illumination, both in intensity and over a finite angular spectrum, is ensured using an imaging homogenizer setup featuring a microoptical Koehler integrator as mixing element [3,4], see Fig. 1.

Replacing the partially coherent $\mathrm{Hg}$ arc lamps in mask aligners with a coherent light source requires the addition of means to the optical system to deal with the effects of coherence. Otherwise Fabry-Perot responses from all interfaces in the optical path would be observable in the resist image. This was achieved by introducing

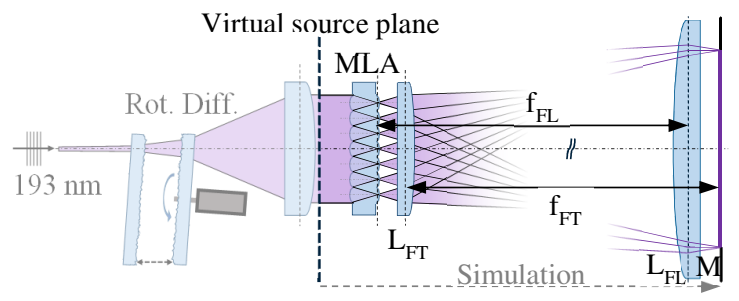

Fig. 1. Mask aligner illumination system for a coherent $193 \mathrm{~nm}$ light source. A diffuser setup featuring a rotating diffuser controls coherence in the mask plane M. An imaging beam homogenizer with microoptical Koehler integrator, consisting of microlens arrays (MLA), and Fourier and Field lenses LFT and $\mathrm{L}_{\mathrm{FL}}$ ensure uniform and telecentric illumination in the mask plane. rotating optical diffusers into the illumination system [2]. While illumination systems relying on rotating diffusers can be modelled by incoherent ray tracing with good results, the investigation of coherence effects in the illumination path requires coherent simulation of the system [5].

Coherent simulation of such systems is demanding: one has to deal with some thousands of microoptical channels in combination with macroscopic length-scales for the overall illumination system and a diffuse yet coherent source. We present an approach using Gaussian beam decomposition (GBD) for modelling coherence in ray tracing simulation as an approach to combine these opposing requirements.

\section{Coherent ray tracing of diffuse sources}

This work was carried out using PHOTON ENGIEERING's FRED ray tracing software, where GBD is implemented.

GBD relies on the approximation of a wavefront by an equally spaced array of Gaussian beamlets. Each one representing the local average intensity and (relative) phase of the wavefront, and propagating in the direction of the local surface normal. Each beamlet consists of a main or base ray and secondary rays which model beamlet waist and divergence. To model the coherent response of an optical system in good approximation these beamlets are coherently summed up on a detector plane after being propagated through the system [6,7].

To test the validity of this approach for simulating illumination systems with optical diffusers, we consider a simple test system: a single lens focusing the wavefront after an ideal optical diffuser illuminated by a plane wave of diameter $D$ onto a detector plane in focal distance $f$ behind the lens, compare Fig. 3 .

The illuminated diffuser is approximated by a GBD plane wave source, where a random phase between 0 and $2 \pi$ is added to each beamlet. On the detector a speckle pattern is visible whose lateral average speckle size $\sigma_{\text {sim }}$

\footnotetext{
* Corresponding author: raoul.kirner@suss.com
} 


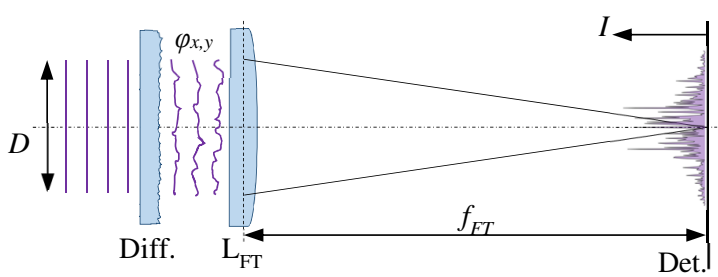

Fig. 3. Layout to test the validity of speckle simulation with GBD. A plane wave of diameter $D$ is shone onto a diffuser where their phase profile $\varphi_{x, y}$ is locally changed. A Fourier lens focuses the wavefront onto a detector in focal distance behind it. There the intensity distribution is captured.

can be found as the width of the autocorrelation peak of said field [8]. We compare this simulated speckle size to the theoretically expected speckle size of an ideal coherent source after an ideal diffuser in focal distance behind an ideal lens, the diffraction limited spot size [9],

$$
\sigma_{x, y} \approx 1.22 \frac{\lambda f}{D} \text {. }
$$

Figure 3 shows this comparison, and furthermore illustrates the robustness of this approach. The approach delivers constant results for a large variety of source sampling rates and source diameters. For $\sigma_{\text {sim }}$ the autocorrelation peak is measured at full width half maximum (FWHM), containing approximately $68 \%$ of the total energy, compared to the expected diffraction limited spot size, containing roughly $84 \%$ of the energy. This leaves a discrepancy of about $4 \%$ to the found mean value for the speckle size ratio $S$ which can be contributed to the noise floor of the simulation.

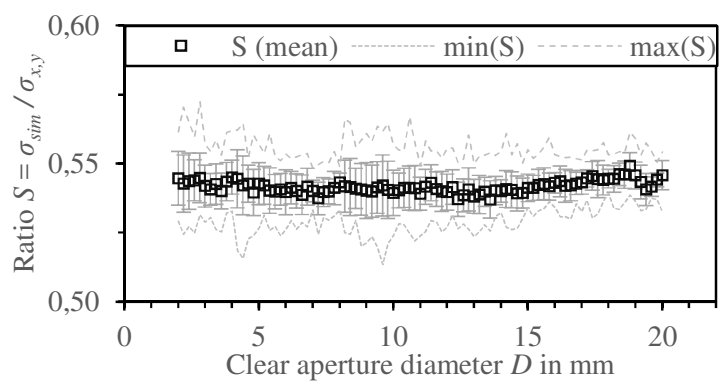

Fig. 2. Ratio $S$ of simulated FWHM speckle size $\sigma_{\text {sim }}$ to theoretical lateral speckle $\sigma_{x, y}$ size as function of beam diameter $D$. Error bars show the standard deviation of variable source sampling (10...250 $\lambda$ beamlet spacing). Dashed lines show local minimum and maximum values of $S$.

\section{Simulation of a multi-aperture system}

To coherently simulate an imaging homogenizer setup the path before the MLA is replaced by a randomized GBD source as described in the previous part, compare Fig. 1.

The source sampling has been carefully chosen to allow for successful implementation: deletion of beamlets has to be avoided by ensuring that all rays of each beamlet always pass the same surfaces (one condition of GBD). This is critical when modelling multi-aperture elements like MLA to maintain correct optical power transmission.

Fig. 4 a) shows the simulated intensity distribution for one random source. Temporal averaging, as implemented with a rotating diffuser in combination with the integrating nature of photoresist as sensor, can be
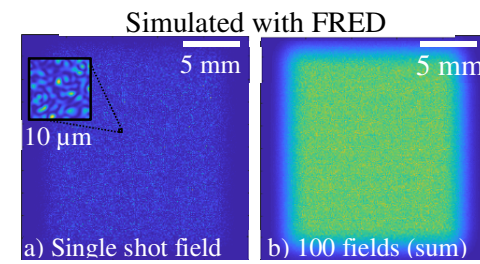

Measured [10]

Fig. 4. a) Simulated static speckle field of a random input source after the beam-shaping system as shown in Fig. 1. b) Superposition of 100 random speckle fields in the mask plane. c) Comparison to a measurement of the same setup, taken with a Silicon photodiode with $300 \mu \mathrm{m}$ square aperture.

modelled by incoherently superimposing multiple simulation runs (static images) with different random initial optical path length distributions added to the source beamlets, as shown in Fig. 4 b). For comparison, Fig. 4 c) shows a measurement of this illumination system.

\section{Conclusion}

We have found conditions under which Gaussian beam decomposition is a valid method to simulate beamshaping systems with microoptical multi-aperture elements. We discussed how to simulate a randomized source in the ray tracing software FRED. We utilized this approach to investigate beam-shaping systems featuring multi-aperture elements for mask aligner lithography in the DUV with a $193 \mathrm{~nm} \mathrm{CW}$ laser light source including diffusers for coherence control.

The authors would like to thank Andreas Vetter and TOPTICA PHOTONICS for the close cooperation.

\section{References}

1. P. Rai-Choudhury, ed., Handbook of microlithography, micromachining, and microfabrication, Volume 1 (SPIE Press, 1997)

2. R. Kirner, A. Vetter, D. Opalevs, C. Gilfert, M. Scholz, P. Leisching, T. Scharf, W. Noell, C. Rockstuhl, R. Voelkel, Opt. Express 26(2), 730 (2018)

3. R. Völkel, U. Vogler, A. Bich, P. Pernet, K. J.Weible, M. Hornung, R. Zoberbier, E. Cullmann, L. Stürzebecher, T. Harzendorf, U. D. Zeitner, Opt. Express 18, 20968-20978 (2010)

4. F. M. Dickey, ed., Laser beam shaping: Theory and Techniques 2nd Ed., chap. 10 (CRC Press, 2017)

5. R. Völkel, K. J. Weible: Proc. SPIE 7102, 1020J (2008)

6. J. Arnaud, Appl. Opt. 24, 538-543 (1985)

7. A. W. Greynolds, Proc. SPIE 0679 (1986)

8. J. W. Goodman, Statistical Optics 2 nd Ed., chap. 7.7 (Wiley, 2015)

9. M. Sjödahl, L. R. Benckert, Appl. Opt. 33(31), 74617471 (1994)

10. R. Kirner, A. Vetter, D. Opalevs, M. Scholz, P. Leisching, T. Scharf, W. Noell, C. Rockstuhl, R. Voelkel, Proc. SPIE 10587, 105870W (2018) 